\title{
A Critical Assessment of Uremia Research
}

\author{
Francesco Locatelli Salvatore Di Filippo Pietro Pozzoni \\ Department of Nephrology and Dialysis, A. Manzoni Hospital, Lecco, Italy
}

\section{Key Words}

Uremia research $\cdot$ On-line hemodiafiltration $\cdot$ High-flux dialysis

\begin{abstract}
There are considerably fewer randomized controlled trials investigating hemodialysis (HD) than other fields of internal medicine, and no significant improvements have been observed over time. Only the National Cooperative Dialysis Study and the HEMO trial were based on hard endpoints such as morbidity and mortality, but neither considered on-line hemodiafiltration or super-flux membranes, which are thought to provide a number of advantages in terms of the cardiovascular condition of uremic patients. However, results of well-designed clinical trials showing that increasing convection may improve the clinical outcome of HD patients are still lacking. The need for maximizing removal of uremic toxins calls for more frequent HD sessions, but this may be affected by many organizational problems. Therefore, well-designed, long-term clinical trials are urgently needed to investigate which currently available therapeutic instruments can improve the clinical outcome of uremic patients.
\end{abstract}

Copyright (C) 2006 S. Karger AG, Basel

\section{Introduction}

As recently pointed out in a systematic review of the scientific literature, one of the main problems affecting nephrologic research is that considerably fewer randomized and controlled trials are published in the field of nephrology than in other internal medicine specialties, and no significant improvement has been observed over time [1]. This is also true of hemodialysis (HD), for which the proportion of published randomized and controlled trials, and their related citations versus total citations, is in line with that of the other specific areas of nephrology considered as a whole [1]. In this context, the extreme paucity of randomized, controlled trials involving uremic patients on chronic HD considering 'hard' endpoints such as morbidity and mortality is hardly surprising, and it is important to remember that dialysis per se has never been tested in a prospective, randomized and controlled trial. Only the National Cooperative Dialysis Study (NCDS) [2] and the more recent (and also more methodologically relevant) Hemodialysis (HEMO) study [3] have considered these characteristics.

\section{Results and Limitations of the NCDS and HEMO Study}

The main finding of the NCDS, a cornerstone in the history of HD clinical research, was the importance of the adequate removal of small solutes (an equilibrated

\begin{tabular}{ll}
\hline KARGER & ( ) 2006 S. Karger AG, Basel \\
0253-5068/06/0241-0071\$23.50/0 \\
$\begin{array}{l}\text { Fax +4161306 } 1234 \\
\begin{array}{l}\text { E-Mail karger@karger.ch } \\
\text { www.karger.com }\end{array}\end{array}$ & $\begin{array}{l}\text { Accessible online at: } \\
\text { www.karger.com/bpu }\end{array}$
\end{tabular}

Prof. Francesco Locatelli, MD, FRCP

Department of Nephrology and Dialysis, Ospedale A. Manzoni

Via Dell'Eremo 9-11

IT-23900 Lecco (Italy)

Tel. +390341 489862, Fax +390341 489860, E-Mail nefrologia@ospedale.lecco.it 
$\mathrm{Kt} / \mathrm{V}$ of approximately 1.0 ), given that lower $\mathrm{Kt} / \mathrm{V}$ values (approaching 0.6) were associated with a significant increase in hospitalization, morbidity and negative outcomes. In terms of 'dialysis time' (analyzed as a mirror of the removal of middle molecules), it is likely that increasing mean dialysis duration from 3 to $4 \mathrm{~h}$ did not offer a benefit that could be detected by such a relatively small study (162 patients) with a follow-up period of only 6 months.

However, it can be said that far from optimal morbidity and mortality results are obtained using low-flux membranes during 'standard' thrice-weekly HD sessions of 3-4 h each. Starting with the hypothesis that the inadequacy of 'standard' HD was attributable to the inadequate removal of middle molecules, HD research over the last 20 years has concentrated on improving the clearance of middle molecules by using low-flux membranes with increasingly larger surfaces ('high-efficiency' HD) or membranes with increasingly greater hydraulic permeability ('high-flux' HD).

The more recent HEMO study used high-efficiency $\mathrm{HD}$ to provide a high $\mathrm{Kt} / \mathrm{V}$ in a short time and high-flux HD to increase the removal of middle molecules; it found a non-significant $8 \%$ reduction in mortality for high-flux HD and a non-significant $4 \%$ reduction in mortality for a higher Kt/V. Possibly, these certainly disappointing results were at least partially due to the design of the study which, for example, selected survivors by enrolling prevalent rather than incident patients, who may have been more suitable for detecting any differences between the two HD techniques. Another major limitation of the study design was the reuse of high-flux dialyzers, as a recent analysis by Lowrie et al. [4] has documented an association between dialyzer reuse and mortality. Finally, as cardiovascular disease is known to be the main cause of mortality in patients on renal replacement treatment, it is a pity that the HEMO study (designed to evaluate whether the high rate of mortality of HD patients may be influenced by the entity and quality of uremic toxin removal) did not consider the use of high-flux membranes in what is currently thought to be the most efficient dialytic technique: on-line hemodiafiltration (HDF). Another important point in this regard is that no use was made of 'really' high-flux membranes capable of removing protein-bound uremic solutes, as well as free water-soluble low-molecular-weight solutes and middle molecules ('super-flux' HD). The results of recent studies suggest that both HDF and super-flux HD can provide a number of advantages in terms of the cardiovascular condition of uremic patients and possible positive effects on their long-term clinical outcome, but these findings need to be tested in large-scale, prospective and randomized clinical trials with an adequate follow-up.

\section{Potential Cardiovascular Benefits of HDF and Super-Flux HD}

Hyperphosphatemia is increasingly recognized as a major risk factor for overall and cardiovascular mortality in HD patients [5, 6]. Zehnder et al. [7] reported significantly greater phosphate clearance with HDF than with high-flux HD, thus suggesting that HDF should be used in patients whose predialysis serum phosphorus levels are difficult to control. However, this was a short (1-week) study that provided no information concerning the longterm predialysis serum phosphorus levels of patients treated with the two techniques.

Another important point is the correction of anemia, as a close relationship has been clearly documented between hemoglobin levels and both survival and the risk of cardiovascular events in HD patients [8]. In a study comparing on-line HDF and a 'soft' form of HDF (similar to high-flux HD in terms of total ultrafiltration), Maduell et al. [9] found significantly higher hemoglobin and lower erythropoietin doses with on-line HDF. However, it is questionable whether improved anemia control is specifically related to HDF and therefore attributable to increased convection, as Schiffl et al. [10] reported similar results in low-flux HD patients treated with an 'ultrapure' rather than a 'commercial' dialysate, and interpreted this as a consequence of the achieved reduction in the patients' chronic inflammatory status.

Improving dialytic tolerance is of paramount importance. A retrospective analysis [11] has found that HDF can lead to a lower requirement for saline solutions to treat hypotensive episodes than low-flux HD, thus suggesting that it may allow the achievement of lower postdialysis body weight levels. It is believed that this in turn can lead to a better control of hypertension, which is volume dependent in most HD patients and another major risk factor for cardiac disease. The greater cardiovascular stability achievable with HDF has also been suggested with respect to high-flux HD in a randomized clinical trial that showed a significant reduction in the number of dialytic sessions complicated by hypotension during HDF [12]. However, it must be pointed out that both studies recorded higher predialysis plasma sodium levels during HDF, and so it can be hypothesized that the improved cardiovascular stability observed with HDF may 
be secondary to lower dialytic sodium removal, with subsequent negative effects on the patient's hypertensive status. Other authors question whether the greater cardiovascular stability is specifically attributable to the high levels of convection guaranteed by HDF. Two studies have found that HDF (both in pre-dilution and post-dilution form) and HD lead to similar cardiovascular tolerance and hemodynamic effects if the two techniques are used in such a way as to achieve the same thermal balance, thus suggesting that the greater reduction in blood temperature within the extracorporeal circuit during HDF may also play an important role $[13,14]$.

The increased removal of middle molecules may be beneficial in terms of morbidity and mortality because uremia per se is another potentially important risk factor for cardiovascular disease. In a randomized clinical trial comparing low-flux HD (with ultrapure dialysate) and HDF with maximized efficiency (60 liters of reinfusate per session), Wizemann et al. [15], confirming previous findings by Locatelli et al. [16], found a $40 \%$ reduction in predialysis plasma $\beta_{2}$-microglobulin concentrations during HDF, whereas the same concentrations remained stable during low-flux HD as expected. On the basis of these findings, the authors suggested the first-choice use of HDF in patients who are expected to require prolonged renal replacement treatment but, although confirming the increased intradialytic reduction in plasma $\beta_{2}$-microglobulin concentrations with HDF, another randomized clinical trial comparing HDF and high-flux HD found that the two groups showed a similar reduction in predialysis concentrations during the 12 months of the study, probably because neither technique is capable of easily removing $\beta_{2}$-microglobulin from the interstitial compartment [17].

Among the 'emerging' cardiovascular risk factors, attention is mainly focused on chronic inflammation, oxidative stress, and hyperhomocysteinemia. It has been shown that high-flux HD with 'commercial' dialysate does not have a positive impact on either chronic inflammation (assessed by means of plasma interleukin-6, interleukin-10 and C-reactive protein concentrations) or oxidative stress (evaluated by determining carbonylic content) [18], although another study found that HDF seemed to control oxidative stress - evaluated by means of predialysis serum advanced glycation end product (AGE) levels - better than both low-flux and high-flux HD, probably because of its greater removal of AGEs [19]. However, the observation of non-significant differences in AGE levels between HDF and high-flux HD using ultrapure dialysate suggests that factors other than increased dialytic removal may contribute to determining the reduced AGE levels with HDF [20].

Although the molecular weight of homocysteine is similar to that of creatinine, it has been reported that the intradialytic reduction in plasma homocysteine levels is only $28 \%$ during low-flux HD [21]. Given that the distribution volume of homocysteine is also comparable with that of creatinine [22], the only possible explanation for this poor level of removal is that a fraction of circulating homocysteine is bound to plasma proteins. Therefore, it is not surprising that high-flux HD is not particularly better than low-flux HD in controlling hyperhomocysteinemia [23], and that a significant reduction in predialysis plasma homocysteine levels can only be achieved by using super-flux dialyzers that are also capable of removing the protein-bound fraction of homocysteine [24]. Furthermore, it has recently been reported that protein-leaking dialyzers improve some inflammatory markers in chronic HD patients [25].

In conclusion, it seems unlikely that increased convection significantly improves the 'depurative' status of patients on renal replacement treatment, at least for as long as standard HD is performed thrice weekly with an overall duration of between 9 and $12 \mathrm{~h}$. This is also supported by the results of two randomized clinical trials, which failed to show any advantages in patient morbidity and mortality in favor of highly convective techniques [15, 16], although the results of still ongoing clinical trials investigating the effect of these techniques on hard endpoints are awaited with much interest [26, 27]. Therefore, more substantial changes in HD prescription may be needed and, if the basic concept is that increasing the dialysis dose allows the greater removal of uremic toxins, the demand for a better control of chronic uremia could be best satisfied by increasing the frequency of HD sessions.

\section{Is Daily Dialysis the Right Solution?}

For the above mentioned, there is now renewed interest in 'daily' HD, be it in 'short high-efficiency' (SDHD) or 'long nocturnal' (NHD) form. These two techniques share more frequent sessions (usually six per week) than standard HD, but they cannot be considered equivalent because NHD sessions are 6-8 $\mathrm{h}$ and SDHD sessions $1.5-$ $2.5 \mathrm{~h}$. Consequently, the former leads to much greater removal of middle molecules (which is time dependent), although the removal of low-molecular-weight solutes may be similar provided that blood flow rate, dialysis flow 
rate and dialyzer surface are lower in NHD than in SDHD. However, mean predialysis plasma urea concentrations have been reported to be lower with both techniques than in conventional HD because of the higher 'standard' Kt/V delivered by daily HD [28].

Although there are still no data showing that daily HD is more beneficial in terms of patient morbidity and mortality than standard HD, a number of studies have found a beneficial effect on many clinical parameters that may positively affect clinical outcome. The London Daily/ Nocturnal Study showed that patients treated with SDHD or NHD had significantly lower mean arterial pressure (MAP), and therefore, required less use of antihypertensive medications than those on standard HD, whose MAP remained unchanged after 18 months of follow-up [29]. However, the reduced MAP in the NHD patients was not accompanied by a decrease in total body water, thus suggesting the possible contribution of other mechanisms in achieving better blood pressure control.

The same study found that serum phosphorus levels and use of phosphate binders were not significantly affected by SDHD, whereas the end-of-study predialysis serum phosphorus levels in the patients treated with NHD were significantly lower than those obtained with SDHD or standard HD, and also allowed much lower doses of phosphate binders, particularly calcium carbonate [30]. However, one effect of this reduced calcium intake, together with the use of low dialysate calcium levels $\left(\mathrm{Ca}^{2+} 1.25 \mathrm{mmol} / \mathrm{l}\right)$, was a significant increase in serum parathyroid hormone levels, which was probably induced by the development of a negative calcium balance. These results raise some questions concerning the appropriateness of decreasing calcium in the dialysate, with the final aim of decreasing serum calcium according to Block et al. [5], given that the recent availability of non-calcium-containing phosphate binders (which are suggested as firstchoice therapy in current clinical guidelines) will probably decrease the use of calcium-based binders - and therefore calcium intake - during the interdialytic period; in the near future, the use of calcimimetics will add to the problem.

In terms of anemia control, the NHD patients in the London Daily/Nocturnal Study showed a significant increase in hemoglobin concentrations from baseline, although no significant between-group differences in predialysis levels were found after 18 months of treatment; furthermore, higher erythropoietin doses were used during NHD, although serum ferritin, transferrin saturation and iron supplementation were comparable in the three groups [31].
A recent comparison of low-flux HD and SDHD showed that the latter was associated with a significant reduction in serum AGEs, which possibly contributed to slowing down the atherosclerotic and amyloidotic processes that occur in patients with chronic uremia [32]; in another study, mean predialysis plasma $\beta_{2}$-microglobulin concentrations nearly halved after 9 months of NHD [33].

Maduell et al. [34] compared thrice-weekly and daily HDF (halving the session time and doubling the number of weekly sessions), and found that daily HDF led to a significant decrease in the occurrence of left ventricular hypertrophy and normalized MAP values (allowing the discontinuation of antihypertensive treatment) in 2 of the 3 patients who were hypertensive at baseline. Standard $\mathrm{Kt} / \mathrm{V}$ increased from 2.6 to 3.9, accompanied by decreasing predialysis concentrations of urea, creatinine, uric acid, $\beta_{2}$-microglobulin and homocysteine; moreover, daily HDF allowed much less use of phosphate binders, and an important nutritional finding was that dry body weight and the protein catabolic rate increased after 6 months of treatment [34]. Similarly, positive results were observed in 5 pediatric patients switched from thrice-weekly to daily HDF (with an overall duration of 12 and $18 \mathrm{~h}$, respectively), with a significant decrease in left ventricular hypertrophy, MAP and serum phosphorus levels, and an increase in caloric intake and body mass index after 12 months [35].

Although daily HD seems to be associated with an improved biochemical profile and better blood pressure control, there is still no evidence of longer survival in comparison with thrice-weekly HD, and there is some concern about the quality of life. It must also be stressed that most of the studies in favor of daily HD are monocenter, small and uncontrolled studies, and a recent analysis of the International Quotidian Dialysis Registry showed that 78 patients treated with daily HD in Northern America were much younger and had a much lower prevalence of diabetes and cardiac disease than those on standard HD [36].

Although the HEMO study did not provide an answer as to whether substantially increasing the dialysis dose (as with daily HD) can reduce morbidity and mortality, it did show that maximizing it with the current thrice-weekly schedule does not lead to any significant advantages in terms of mortality, thus suggesting the need for newer approaches. Many argue that the benefits of daily HD are so evident that no further studies (including randomized clinical trials) are necessary, but others insist on verifying them by means of more rigorous methods of clinical eval- 
uation. However, it can also be questioned whether daily treatment can actually be proposed and applied to most HD patients and why daily HD remains a 'niche' treatment many years after the publication of its first outstanding results. The most likely answer to this second question seems to be that its widespread use is heavily conditioned by organizational difficulties, the need for the constant presence of the partner in home HD, and vascular accessrelated problems.

\section{Conclusions}

In the evolving scenario of dialysis patients and techniques, and the still very small number of well-designed clinical studies on HD, there is an urgent need for long- term clinical trials of the therapeutic instruments currently available to improve clinical outcomes. In particular, these should consider the clinical impact of innovative techniques, such as on-line HDF, super-flux dialyzers and daily dialysis, and various aspects of HD prescription, such as dialysate calcium concentrations in an era of non-calcium-containing phosphate binders, non-hypercalcemic vitamin D analogues and calcimimetics. However, in order to provide results that can be translated into clinical practice, such studies need extended observation periods and a sufficient number of patients and must be adequately controlled for some crucial aspects of HD treatment (above all, the dialysis dose and the quality of dialysis fluid) while simultaneously adjusting for patient characteristics and comorbidities.

\section{References}

-1 Strippoli GF, Craig JC, Schena FP: The number, quality, and coverage of randomized controlled trials in nephrology. J Am Soc Nephrol 2004; 15:411-419.

-2 Lowrie EG, Laird NM, Parker TF, et al: Effect of the hemodialysis prescription on patient morbidity: report from the National Cooperative Dialysis Study. N Engl J Med 1981;305: 1176-1181.

3 Eknoyan G, Beck GJ, Cheung AK, et al: Effect of dialysis dose and membrane flux in maintenance hemodialysis. N Engl J Med 2002;347: 2010-2019

4 Lowrie EG, Li Z, Ofsthun N, et al: Reprocessing dialysers for multiple uses: recent analysis of death risks for patients. Nephrol Dial Transplant 2004; 19:2823-2830.

$\checkmark 5$ Block GA, Klassen PS, Lazarus JM, et al: Mineral metabolism, mortality, and morbidity in maintenance hemodialysis. J Am Soc Nephrol 2004; 15:2208-2218.

-6 Young EW, Albert JM, Satayathum S, et al: Predictors and consequences of altered mineral metabolism: The Dialysis Outcomes and Practice Patterns Study. Kidney Int 2005;67: 1179-1187.

7 Zehnder C, Gutzwiller JP, Renggli K: Hemodiafiltration - A new treatment option for hyperphosphatemia in hemodialysis patients. Clin Nephrol 1999;52:152-159.

8 Locatelli F, Pisoni RL, Combe C, et al: Anemia in haemodialysis patients of five European countries: association with morbidity and mortality in the Dialysis Outcomes and Practice Patterns Study (DOPPS). Nephrol Dial Transplant 2004; 19:121-132.

$\checkmark 9$ Maduell F, del Pozo C, Garcia H, et al: Change from conventional hemodiafiltration to on-line hemodiafiltration. Nephrol Dial Transplant 1999; 14:1202-1207.
10 Schiffl H, Lang SM, Bergner A: Ultrapure dialysate reduces dose of recombinant human erythropoietin. Nephron 1999;83:278-279.

11 Pizzarelli F, Cerrai T, Dattolo P, et al: Convective treatments with on-line production of replacement fluid: a clinical experience lasting 6 years. Nephrol Dial Transplant 1998;13:363369

12 Lin CL, Huang CC, Chiang CT, et al: Clinical improvement by increased frequency of online hemodiafiltration. Renal Failure 2001;23 193-206.

13 Donauer J, Schweiger C, Rumberger B, et al: Reduction of hypotensive side effects during online-hemodiafiltration and low temperature hemodialysis. Nephrol Dial Transplant 2003; 18:1616-1622.

14 Karamperis N, Sloth E, Jensen JD: Predilution hemodiafiltration displays no hemodynamic advantage over low-flux hemodialysis under matched conditions. Kidney Int 2005;67 1601-1608.

15 Wizemann V, Lotz C, Techert F, Uthoff S: Online hemodiafiltration versus low-flux hemodialysis. A prospective randomized study. Nephrol Dial Transplant 2000;15(suppl 1):4348.

16 Locatelli F, Mastrangelo F, Redaelli B, et al: Effects of different membranes and dialysis technologies on patient treatment tolerance and nutrition parameters. Kidney Int 1996;50: 1293-1302.

17 Ward RA, Schmidt B, Hullin J, et al: A comparision of on-line hemodiafiltration and highflux hemodialysis: a prospective clinical study. J Am Soc Nephrol 2000;11:2344-2350.
18 Pupim LB, Himmelfarb J, McMonagle E, et al: Influence of initiation of maintenance hemodialysis on biomarkers of inflammation and oxidative stress. Kidney Int 2004;65:23712379.

19 Lin CL, Huang CC, Yu CC, et al: Reduction of advanced glycation end product levels by online hemodiafiltration in long-term hemodialysis patients. Am J Kidney Dis 2003;42:524531.

20 Genderman A, Wagner Z, Solf A, et al: Plasma levels of advanced glycation end products during haemodialysis, haemodiafiltration and haemofiltration: potential importance of dialysate quality. Nephrol Dial Transplant 2002;17: 1045-1049.

-21 Arnadottir M, Berg AL, Hegbrant J, Hultberg $\mathrm{B}$ : Influence of haemodialysis on plasma total homocysteine concentration. Nephrol Dial Transplant 1999; 14:142-146.

22 Guttormsen AB, Mansoor AM, Fiskerstrand $\mathrm{T}$, et al: Kinetics of plasma homocysteine in healthy subjects after peroral homocysteine loading. Clin Chem 1993;39:1390-1397.

23 House AA, Wells GA, Donnelly JG, et al: Randomized trial of high-flux vs low-flux haemodialysis: effects on homocysteine and lipids. Nephrol Dial Transplant 2000;15:1029_ 1034.

24 Van Tellingen A, Grooteman MPC, Bartels $\mathrm{PCM}$, et al: Long-term reduction of plasma homocysteine levels by super-flux dialyzers in hemodialysis patients. Kidney Int 2001;59:342347.

25 Galli F, Benedetti S, Floridi A, et al: Glycoxidation and inflammatory markers in patients on treatment with PMMA-based protein-leaking dialyzers. Kidney Int 2005;67:750-759. 
26 Bolasco P, Altieri P, Andrulli S, et al: Convection versus diffusion in dialysis: an Italian prospective multicentre study. Nephrol Dial Transplant 2003;18(suppl 7):50-54.

27 Penne EL, Blankestijn PJ, Bots ML, et al: Effect of increased convective clearance by online hemodiafiltration on all cause and cardiovascular mortality in chronic hemodialysis patients - the Dutch CONvective TRAnsport Study (CONTRAST): rationale and design of a randomised controlled trial. Curr Control Trials Cardiovasc Med 2005;6:8.

28 Gotch FA, Levin NW: Daily dialysis: the long and the short of it. Blood Purif 2003;21:271281 .
29 Nesrallah G, Suri R, Moist L, et al: Volume control and blood pressure management in patients undergoing quotidian hemodialysis. Am J Kidney Dis 2003;42(suppl 1):S13-S17.

30 Lindsay RM, Alhejaili F, Nesrallah G, et al: Calcium and phosphate balance with quotidian hemodialysis. Am J Kidney Dis 2003;42 (suppl 1):S24-S29.

31 Rao M, Muirhead N, Klarenbach S, et al: Management of anemia with quotidian hemodialysis. Am J Kidney Dis 2003;42(suppl 1):S18S23.

32 Floridi A, Antolini F, Galli F, et al: Daily haemodialysis improves indices of protein glycation. Nephrol Dial Transplant 2002;17:871878
33 Pierratos A: Daily hemodialysis: an update. Curr Opin Nephrol Hypertens 2002;11:165171 .

34 Maduell F, Navarro V, Torregrosa E, et al: Change from three times a week on-line hemodiafiltration to short daily on-line hemodiafiltration. Kidney Int 2003;64:305-313.

35 Fischbach M, Terzic J, Laugel V, et al: Daily on-line haemodiafiltration: a pilot trial in children. Nephrol Dial Transplant 2004; 19:23602367.

36 Nesrallah G, Garg AX, Moist LM, et al: The international quotidian dialysis registry: annual report 2005. Hemodialysis Int 2005;9: 203-209. 